\title{
UNA METODOLOGÍA CUALITATIVA PARA EL ESTUDIO DE LAS INFLUENCIAS AFECTIVAS EN EL CONOCIMIENTO DE LAS MATEMÁTICAS
}

\author{
GÓMEZ CHACÓN, INÉS M. \\ Departamento de Didáctica de las Matemáticas. \\ Instituto de Estudios Pedagógicos Somosaguas. Madrid.
}

\section{SUMMARY}

The aim of the investigation that sustains the methodology exposed on this paper is to determine and to describe the dynamic of interaction between the cognitive and affective factors on the mathematics learning in young people with failure at school and in a context of social exclusion.

The research has a qualitative orientation because it is a descriptive-interpretative study about the interrelations between cognition and affect. To typify this interrelation a holistic perspective has been adopted. A design has been developed combining specific ethnographic techniques with those of cases studies and reflection about the action. In this paper we explain the methodology aspects. It describes the elaboration and attributes of two instruments specially designed for the study: one to investigate the beliefs and the other to make a diagnose of the emotional reactions.

\section{INTRODUCCIÓN}

Durante la década de los ochenta, los estudios realizados con metodologías cualitativas en didáctica de las matemáticas experimentan un incremento considerable (Eisenhart, 1988, Schoenfeld, 1994). Entre los factores que más influyeron en este cambio metodológico podemos citar: la apertura epistemológica y la apertura interdisciplinar que experimentó la investigación en la enseñanzaaprendizaje de las matemáticas.

Entre las líneas de investigación que más han contribuido al incremento de los estudios cualitativos destaca la que se orienta, de manera muy diversificada, al estudio de la estructura cognitiva de los alumnos, al estudio sobre las estrategias usadas por los estudiantes en resolución de problema, o al estudio sobre metacognición y creencias.
Dentro de la investigación escolar, la comprensión del aprendizaje se ha medido por los logros académicos de los aspectos cognitivos. Aun reconociendo que los resultados afectivos, procedentes de la metacognición y dimensión afectiva del individuo, determinan la calidad del aprendizaje, a menudo estos estudios se han dejado de lado. A finales de la década de los ochenta gran parte de las investigaciones en didáctica de las matemáticas sobre los procesos de aprendizaje ha comenzado a centrarse en estos aspectos, a los que se añade la importancia del contexto sociocultural en el aprendizaje de la matemática (D’Ambrosio, 1985; Bishop, 1988; Mellin-Olsen 1987; Lerman, 1996). Este nuevo enfoque de la dimensión afectiva, auspiciado en gran medida por los trabajos del educador matemático McLeod (1988, 1992, 1994)른 pone de manifiesto que las cuestiones afectivas juegan 
un papel esencial en la enseñanza y aprendizaje de la matemática, y algunas de ellas están fuertemente arraigadas en el sujeto y no son fácilmente desplazables por la instrucción.

Si son escasos los estudios sobre la dimensión afectiva y el aprendizaje de la matemática, como indicábamos, aún más raros son los relativos al estudio de la emoción. Las razones que parecen haber frenado a éstos son, por una parte, la gran dificultad de su diagnóstico al no disponer de instrumentos adecuados para ello y, por otra, la dificultad de ubicarlos en un marco teórico.

«La falta de atención a la emoción es probablemente debida al hecho de que la investigación en cuestiones afectivas -en su mayor parte- ha buscado factores actitudinales que son estables y que se pueden medir mediante cuestionarios. No obstante, ha habido estudios dirigidos a los procesos involucrados en el aprendizaje de la matemática, y alguno de ellos ha prestado atención a las emociones... Sin embargo, nunca ha jugado un papel relevante en las investigaciones sobre el dominio afectivo en matemáticas. El mayor problema ha sido la falta de un marco teórico dentro del cual interpretar el rol de las emociones en el aprendizaje de la matemática. La teoría de Mandler puede ser un buen punto de partida para construir ese marco teórico...» (McLeod, 1990, p. 21).

Por tanto, teniendo en cuenta la relevancia de estos estudios y los distintos elementos que no han hecho fácil su desarrollo, en este artículo describiremos la metodología utilizada en nuestro trabajo sobre dimensión afectiva y aprendizaje de la matemática. Prestaremos especial atención a algunos de los instrumentos elaborados para el diagnóstico de reacciones emocionales y creencias acerca de las matemáticas (Gómez-Chacón, 1997). Comenzaremos señalando brevemente las finalidades del mismo.

\section{LA FINALIDAD DEL TRABAJO}

El trabajo al que corresponde la metodología que vamos a exponer se encuadra en el marco de las áreas de la psicología y sociología en educación matemática, preocupadas por los aspectos que favorecen o bloquean el aprendizaje de esta disciplina.

Las dificultades en la enseñanza y el aprendizaje de la matemática, que se abordan en este estudio, se refieren a las originadas por los factores afectivos y culturales o de contexto, más específicamente a las emociones (reacción emocional) y creencias acerca de la matemática. La finalidad general del trabajo se puede formular del modo siguiente: determinar y describir la dinámica de interacción entre los factores cognitivos y afectivos en el aprendizaje de la matemática en poblaciones de fracaso escolar y en contextos de exclusión social.

A esta finalidad se le dio una forma más operativa para guiar el diseño de la investigación. Siendo nuestro estu- dio de carácter fundamentalmente descriptivo-interpretativo no nos planteamos hipótesis de trabajo, sino que señalamos un objetivo a cubrir por la investigación y delimitamos unas cuestiones centrales a las que el trabajo debería dar respuesta. Las hipótesis, en su caso, surgirían como resultado del estudio y abrirían nuevas perspectivas de investigación, como así fue.

Se han indagado las reacciones emocionales de los individuos a la luz del contexto sociocultural de la práctica, donde cognición y afecto se entretejen.

En el estudio se ha tratado de:

- establecer y describir las relaciones significativas entre cognición y afectividad (afecto local y global, dos constructos definidos en el estudio);

- indagar el origen de estas reacciones afectivas y constatar la evolución de los sujetos (modificaciones, cambios, etc.) después de su participación en un programa de actuación didáctica que integra la dimensión afectiva (metaafecto); y

- analizar si se podrían interpretar las reacciones emocionales de los jóvenes desde la perspectiva de la identidad social.

En su planteamiento inicial, la investigación se sitúa en la perspectiva cognitiva (McLeod, 1988, 1992), basada en la aproximación sociocognitiva y en el desarrollo teórico de Mandler $(1984,1989)$ acerca de la emoción (teoría de la discrepancia, aproximación psicológica). Esta teoría proporciona una explicación plausible para entender cómo las creencias de los estudiantes, y sus interacciones en situaciones de resolución de problemas, conducen a respuestas «afectivas-emocionales». Hemos incorporado esta perspectiva como guía para realizar las observaciones de aula, para rastrear las reacciones afectivas desde las creencias y expectativas de las que surgen, para el diseño de algunos instrumentos que recogen información sobre reacciones emocionales y para el diseño de algunas actividades de la intervención; también como posible marco interpretativo, cuando nos aproximamos al estudio de la interacción cognición-afecto en el sujeto a través de escenarios ${ }^{2}$ simples, es decir, cuando el estudiante está involucrado en el proceso de resolución de problemas matemáticos y focalizamos nuestra atención para indagar la interrelación afecto-cognición en fases de resolución de problemas, errores en su resolución, etc.

Sin embargo, buscábamos comprender más allá la experiencia surgida en la clase. Para ello era necesario extender nuestra instancia interpretativa, desarrollando una perspectiva sociológica en la aproximación al afecto en la actividad matemática. Para este propósito hemos tenido como referencia la perspectiva de Cobb y otros (1989), que prestan particular atención al acto emocional y al contexto social a través de la relevancia de la interacción en la construcción de significados (interaccionismo simbólico) y la reflexibidad de la etnometodología. 
Nuestro supuesto básico es que la cultura y los procesos sociales son parte integrante de la actividad matemática y que, para la identificación de las reacciones emocionales, es necesario tener en cuenta cómo la persona está valorando el objeto o la situación. Las emociones tienen un subyacente racional, dentro de la cultura en general y, en particular, en la cultura de la clase de matemáticas. La observación cualitativa de las emociones sería posible describiendo el contexto social en que se producen. Por tanto, el análisis de la emoción no debería restringirse a escenarios simples (errores, fases concretas de resolución de problemas, etc.). El interaccionismo simbólico nos permite ampliar estos escenarios simples a escenarios complejos, ya que es mediante un proceso creativo cómo el actor construye su afectividad en relación con la matemática; la definición de la situación e interpretación del actor social son esenciales para comprender su conducta. Las emociones no serían respuestas automáticas o consecuencias de activaciones fisiológicas, sino que serían el resultado complejo del aprendizaje, de la influencia social y de la interpretación. En la interacción social, las emociones jugarán un papel fundamental para el establecimiento de relaciones de pertenencia social y de estatus social. Consideramos la identidad social como polo organizador que moviliza el conjunto de las reacciones afectivas de cada sujeto hacia la matemática y su aprendizaje. La identidad social del individuo configura la estructura local y global del afecto en matemáticas.

\section{ESTRUCTURA GENERAL DE LA INVES- TIGACIÓN}

El objetivo que se planteó requirió la realización de dos estudios interdependientes de carácter etnográfico: uno exploratorio y otro principal. El primero se realizó en el curso 1993-94 con 70 chicos/as de cinco centros-taller (públicos y privados), ubicados en distintas zonas periféricas de Madrid (norte, sur, sureste), caracterizadas por la desventaja sociocultural y con rasgos similares a la población con la que se realizaría el estudio principal. Se seleccionó uno de los centros-taller, como profesora voluntaria de matemáticas se realizó un seguimiento a lo largo de todo el curso. Para la recogida de la información se utilizó un cuestionario y la observación participante. El segundo estudio etnográfico se llevó a cabo en los cursos 1994-95 y 1995-96 con un grupo de estudiantes de uno de los centros-taller-Centro-taller de Fuencarral de la Asociación Norte Joven en el taller de Ebanistería-, el mismo que fue elegido en el exploratorio (23 sujetos del taller de ebanistería).

Para el estudio principal realizamos un diseño en el que se combinan las técnicas propias de la etnografía con las de los estudios de casos, así como la reflexión sobre la propia acción.

La estrategia básica de investigación está basada en estudio de casos en profundidad. Esta elección se hace con el objeto de explorar con los mismos jóvenes variedad de posibles explicaciones que iluminen la interrelación entre cognición y afecto.
En nuestra investigación, el estudio de casos se desarrolla siguiendo una orientación metodológica cualitativa denominada cualitativa etnografía motivada (Abreu, 1993); es decir, que, cuando el investigador comienza su observación etnográfica, ya ha seleccionado el aspecto de la situación social en la que él se quiere centrar, y también su selección está influenciada por sus creencias acerca del fenómeno en el que se ha centrado.

El estudio principal tomó en cuenta los diversos aspectos del estudio exploratorio y, fundamentalmente, aquéllos que ponían de manifiesto la relevancia de las emociones y las creencias en el aprendizaje; la necesidad de «abrir» el estudio (la matemática) a la identidad cultural y de contexto de los estudiantes.

Trabajar desde una perspectiva holística (que tenga en cuenta a la persona en situación) hizo que nos aproximáramos al problema, en su marco teórico, desde varios ámbitos de conocimiento:

- El aprendizaje desde la perspectiva sociocultural: Hemos tenido en cuenta la matemática como conocimiento cultural (Bishop, 1988; D’Ámbrosio, 1985; Nunes, 1992; Stigler y Bares, 1988); el contexto sociocultural y motivacional en el aprendizaje (investigaciones que desarrollan una aproximación sociocultural a la instrucción (Moll, 1992; Civil, 1994); los estudios sobre comunidades de aula, donde la matemática es socialmente construida (Cobb, Wood y Yackel, 1991; Civil, 1994; Schoenfeld, 1991), las investigaciones que documentan las discontinuidades de la matemática en la escuela y en la vida cotidiana (Carraher, Carraher y Schliemann, 1988; Bishop y Abreu, 1991; Lave, 1988); los presupuestos constructivistas en el aprendizaje (Cobb, Yackel y Wood, 1992; Steffe, 1991; Confrey, 1991); etc.

- La dimensión afectiva en la educación matemática: Nos hemos acercado a las distintas aproximaciones teóricas del ámbito de la psicología y de la sociología que están en la base de los desarrollos en educación matemática (McLeod, 1992; Cobb, Yackel y Wood, 1989; Lafortune y St. Pierre, 1994; Goldin, 1988; Nimier, 1988).

- La perspectiva de la identidad social (Camilleri et al., 1990; Lloyd y Duveen, 1992; Abreu 1993).

Consideramos que las teorías cognitivas y las teorías socioculturales pueden unirse en un esfuerzo por crear una teoría comprensiva de la actividad humana (Eisenhart, 1988, p. 10); en nuestro caso, de la dimensión afectiva en matemáticas. Para nosotros es relevante tanto el grupo humano con su cultura, su sistema de comunicación y su estructura institucional (fenómenos de educación matemática considerados prioritariamente sociales y antropológicos) como el plano personal, con los aspectos intraindividuales del conocimiento y de las relaciones psíquicas.

Sugerimos que una integración de estas perspectivas de forma crítica ayuda a comprender la compleja interac- 
Figura 1

Estructura de la investigación.

NIVEL 1: CONTEXTO SOCIOCULTURAL. Describe la construcción y transformación de la representación social del conocimiento matemático del grupo. Se trabaja en este nivel cuando se describe la organización socio-cultural del ámbito de matemática del taller (trabajo) y de la escuela.

NIVEL 2: GRUPO DE INTERACCION TALLER-CLASE DE MATEMATICAS. Describe la evocación, reacción y transformación de la matemática como objeto de saber en interacción social. Se trabaja este nivel cuando se describe las prácticas y reacciones emocionales en la clase de matemáticas y en el taller, y la interacción social.

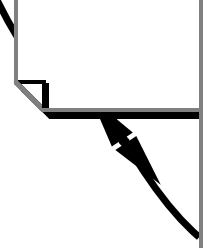

NIVEL 3: SUJETO. Describe el desarrollo del individuo en relación a su mundo y a su identidad social, sus rutas significativas de interacción cognición y afecto al trabajar la matemática.

ción de los factores afectivos, cognitivos y culturales que entran en juego en el aprendizaje de la matemática.

Incorporar esta perspectiva holística nos exigió la búsqueda de una comprensión de las cuestiones de investigación planteadas a tres niveles: nivel del sujeto; nivel micro -sobre las interacciones en el aula y en el taller al trabajar la matemática-; y nivel del contexto social y cultural. La figura 1 hace referencia a cómo conceptualizamos dichas relaciones.

Para la indagación de los datos se hizo un seguimiento diferenciado de estos jóvenes:

a) el conjunto total de jóvenes que durante los cursos 1994-95 y 1995-96 pasan por el taller de ebanistería (23 jóvenes) para indagar datos correspondientes al nivel 1 de la estructura de la investigación;

b) el grupo de jóvenes que permanecen en el curso 199495 en el taller de ebanistería (13 jóvenes) para indagar datos correspondientes al nivel 2 de la estructura de la investigación;

c) un grupo de 7 jóvenes de entre los anteriores, que son los que asisten a las clases de matemáticas, y a quienes realiza un seguimiento más específico para buscar información relativa al nivel 3 .
Más adelante explicitaremos la relación entre los instrumentos y niveles. Para la descripción de la metodología utilizada nos ceñiremos al segundo de los estudios citados.

\section{Tipo de datos requeridos}

Los datos que hace falta explicitar en este segundo estudio se refieren a:

a) los principales factores afectivos que entran en juego en el aprendizaje de la matemática, y a cuándo y por qué aparecen sus reacciones afectivas hacia la matemática y su aprendizaje;

b) datos que dan una descripción del contexto y de los procesos sociales para facilitar la comprensión de las reacciones emocionales de los estudiantes;

c) las reacciones afectivas y los cambios que se producen en el sujeto durante y después del programa de actuación didáctica;

d) las características, tendencias y perfiles que nos permiten conjeturar el afecto global del sujeto;

e) la identidad social de los individuos. 
Los distintos procedimientos de acceso a la realidad han sido triangulación de perspectivas y triangulación con ayuda de integración de información procedente de cuestionarios, entrevistas al alumnado, entrevista semiestructurada de debate grupal, entrevistas al maestro de taller, observación en el aula, notas de campo, grabaciones en audio, ficheros del centro, etc. (Cuadro I). Esta variación de perspectiva tiene como finalidad una comprensión holística de los jóvenes (Eisenhart, 1988).

Como indicábamos anteriormente, las reacciones afectivas consiguen su carácter cualitativo si son contextualizadas en la realidad social que las produce. Por tanto, el análisis de la emoción no se ha restringido únicamente a escenarios simples (errores, fases concretas de resolución de problemas, etc.), sino que se han abarcado escenarios más complejos, que consideran al estudiante en su realidad social y tienen en cuenta, en el autoconcepto del alumno como aprendiz de matemáticas, la valoración y sentimiento que éste tiene como miembro de un grupo social (Cuadro II).

Para hacer el estudio de casos nos hemos centrado, en primer lugar, en «escenarios simples» (reacciones emocionales en resolución de problemas). Para ello se han determinado el origen de las emociones del sujeto de diferentes modos:

a) mediante observación en clase y posterior confirmación en la entrevista;

b) mediante la propia autoevaluación del sujeto (mapa de humor, gráfica emocional).

Como indicábamos al explicitar la finalidad, para operativizar los aspectos de la interacción de los factores afectivos, cognitivos y culturales, que entran en juego en el aprendizaje de la matemática a través de los escena-

\begin{tabular}{|c|c|}
\hline FUENTE & PROCEDIMIENTOS \\
\hline Actividades en clase & $\begin{array}{l}\text { - Observaciones en el aula. Notas de campo } \\
\text { - Grabaciones en audio } \\
\text { - Instrumentos de emoción } \\
\text { - Protocolos de resolución de las actividades matemáticas }\end{array}$ \\
\hline Actividades del taller & $\begin{array}{l}\text { - Observación en el taller. Notas de campo } \\
\text { - Entrevistas semiestructuradas } \\
\text { - Sesiones de aprendizaje } \\
\text { - Conversaciones informales } \\
\text { - Grabaciones en audio } \\
\text { - Artefactos del taller }\end{array}$ \\
\hline Estudiantes & $\begin{array}{l}\text { - Cuatro entrevistas semiestructuradas en profundidad } \\
\text { - Entrevista semiestructurada de debate grupal } \\
\text { - Cuestionario «Eslogan de las clases de matemáticas» } \\
\text { - Instrumentos de emoción } \\
\text { - Contactos informales a lo largo del periodo de investigación }\end{array}$ \\
\hline Maestro del taller & $\begin{array}{l}\text { - Entrevistas informales semiestructuradas } \\
\text { - Sesiones planificadas para el diseño y la validación del material del programa } \\
\text { de actuación didáctica } \\
\text { - Notas de campo }\end{array}$ \\
\hline Educadores y profesores & $\begin{array}{l}\text { - Sesiones planificadas para el diseño y la validación del material del programa } \\
\text { de actuación didáctica } \\
\text { - Conversaciones informales }\end{array}$ \\
\hline Fichero escolar y documentación del centro & $\begin{array}{l}\text { - Historial académico del estudiante } \\
\text { - Informe pedagógico } \\
\text { - Informe de los servicios sociales }\end{array}$ \\
\hline
\end{tabular}


Cuadro II

Escenarios simples y escenarios complejos

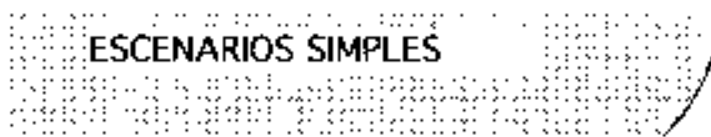

ESTRUCTURA LOCAL DEL AFECTO
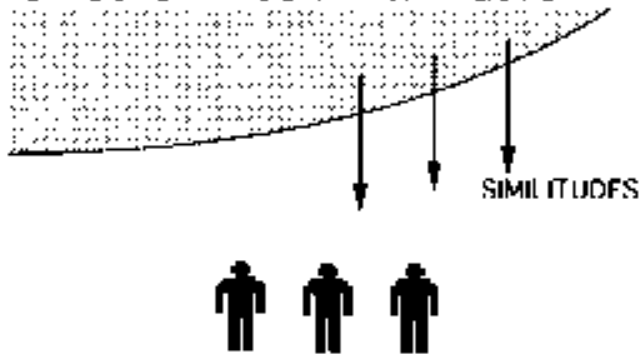

EVOLUCION DIFERENTE

Qué tipo de miembros son y cómo se posicionan en el grupo social

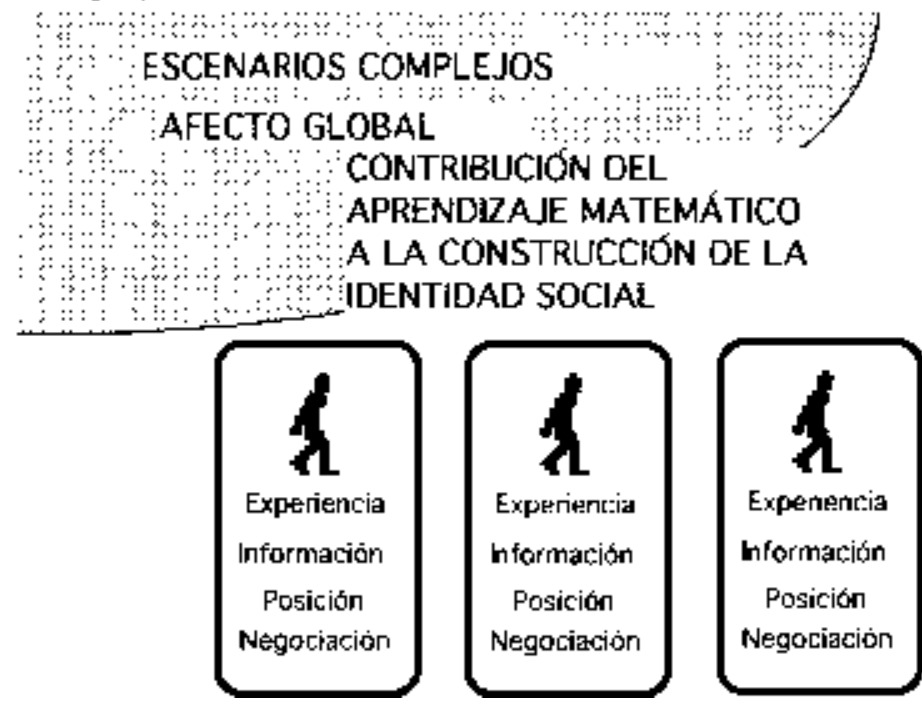

rios simples y complejos, y con objeto de ir generando una teoría comprensiva de la dimensión afectiva del sujeto en matemáticas, se definieron dos constructos: afecto local y afecto global.

El afecto local, es decir, los estados de cambio de sentimientos o reacciones emocionales durante la resolución de una actividad matemática a lo largo de toda la sesión de clase, se ha indagado a través de los escenarios simples. Éstos nos han permitido establecer la estructura local afecto-cognición. Está formada por las relaciones conjeturadas entre las reacciones emocionales y los

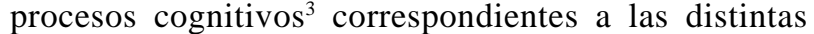
fases en la resolución de la tarea matemática. En este trabajo, se ha elaborado, a partir de los datos recogidos y del análisis del afecto local a lo largo de distintas sesiones de clase y en las entrevistas realizadas al sujeto. La estructura local expresa tipos de interacción cuando el código emocional interactúa con el sistema cognitivo: interrupciones, desviaciones, atajos cognitivos, que se pueden expresar a través de distintas rutas.

El afecto global, entendido como el resultado de las rutas seguidas (en el individuo) en el afecto local que se establecen con el sistema cognitivo que van contribuyendo a la construcción de estructuras generales del concepto de uno mismo ${ }^{4}$ y a las creencias acerca de la matemática y su aprendizaje, se ha indagado a través de los escenarios complejos. Estos contemplan a la persona en su contexto sociocultural y en interacción con los otros. Tienen en cuenta el aprendizaje de la matemática como construcción de la identidad social del joven y 
Figura 2

Relación entre afecto local y afecto global.

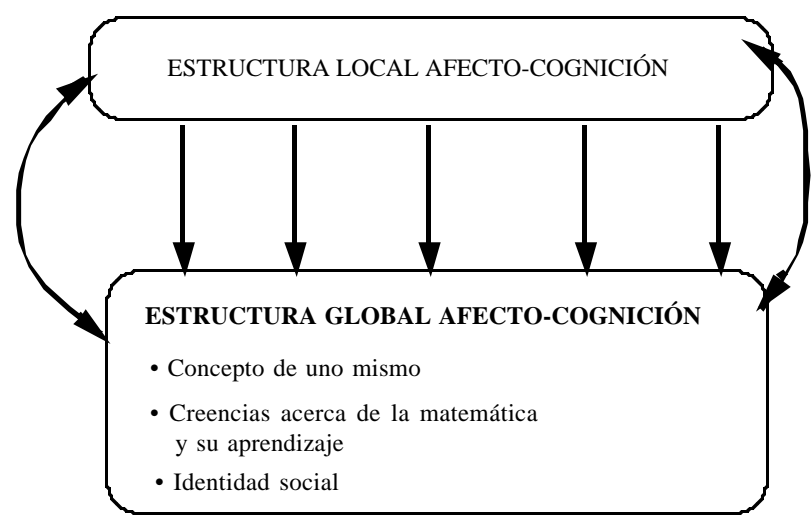

contextualizan las reacciones emocionales en la realidad social que las produce.

Hacemos notar las implicaciones del afecto local en el afecto global y viceversa, como se puede observar en la figura 2, consideramos que el afecto local deja al final un rastro global y la doble dirección del afecto global al local el concepto de uno mismo, las creencias acerca de la matemática y su aprendizaje influye en la determinación de las rutas seguidas en el afecto local. El afecto global configura la estructura local del afecto-cognición en el sujeto.

Igualmente cabe indicar que, al aproximarnos mediante escenarios complejos, contemplamos a la persona en situación (haciendo matemáticas y como actor social), lo que nos permite configurar el afecto global del sujeto. Se obtiene la información de los tres niveles (Fig. 1) para poner de manifiesto el afecto global y local del sujeto.

De estos afectos (y su relación con la cognición) va a tratar el resto del artículo. A continuación pasamos a presentar dos tipos de instrumentos, elaborados en esta investigación, para la recogida de datos relativos a escenarios simples y a escenarios complejos. Estos instrumentos nos han permitido poner de manifiesto la estructura local y global del afecto en los sujetos y ver la implicación entre ambos.

\section{INSTRUMENTOS DE RECOGIDA DE DATOS}

\section{Problemas sobre instrumentos}

Señalamos anteriormente la dificultad de encontrar instrumentos adecuados para medir la dimensión afectiva y dirigidos a estas poblaciones. Hemos elaborado distintos instrumentos expresamente para este trabajo, los cuales han resultado ser una aportación determinante para el mismo. Destacamos algunos de ellos, explicitando la conexión de estos instrumentos con los niveles
(Fig. 1), los escenarios simples y complejos (Cuadro II) y los afectos local y global (Fig. 2):

1) Las entrevistas sobre situaciones. Para recoger datos sobre el sistema de creencias y valores asociado con el conocimiento matemático escolar y sobre el conocimiento matemático en la práctica, en un contexto de desventaja social (nivel 1 y 3 , afecto global, escenario complejo).

2) Mapa de humor de los problemas. Instrumento de diagnóstico de las reacciones emocionales y de autorregulación de la emoción (nivel 2 y 3 , afecto local, escenario simple).

3) Programa de actuación didáctica. Desarrollo de módulos de aprendizaje, orientados a la práctica escolar que integren la dimensión afectiva y el contexto cultural de la práctica (niveles 1, 2, 3; afecto local y global, escenarios simples y complejos).

\section{Entrevistas sobre situaciones para indagar las creencias de los estudiantes}

En el estudio estábamos interesados en rastrear las reacciones emocionales desde las creencias que las originan. Para ello elaboramos dos entrevistas que se realizaban al inicio y al final de la intervención. Estas nos permiten, principalmente, recoger datos correspondientes a los escenarios complejos y al nivel 1 y 3 , para investigar la dimensión global del afecto de los sujetos, sobre todo lo relacionado con los aspectos de identidad social.

A continuación describimos una de ellas, la entrevista I.

\section{Entrevista I (EI)}

Es una entrevista semiestructurada en profundidad sobre situaciones. Es empleada para conocer las ideas (creencias, reacciones, hechos de su experiencia) de los alumnos sobre aspectos o situaciones de la vida diaria; es decir, para recoger datos sobre el sistema de creencias y valores asociado con el conocimiento matemático escolar y sobre el conocimiento matemático en la práctica en un contexto de desventaja social. Estas situaciones se presentan en fotografías. Para cada fotografía se preparan una serie de preguntas indicativas, relativas al uso y éxito de la matemática escolar, a las creencias acerca de la matemática como objeto de conocimiento, orientadas a que el entrevistado describa lo que ve y explique -desde su punto de vista- cómo cree que sucede aquello. A medida que la entrevista progresa se pueden investigar las creencias o reacciones acerca de la matemática que aparecen de modo espontáneo en la conversación del alumno, o que la entrevistadora introduce oportunamente.

El protocolo de esta entrevista contiene preguntas que apenas pueden responderse con opiniones preconcebidas; por ejemplo, se muestran fotos en las que se plantea a los jóvenes que sugieran perspectivas pensando en los amigos; o se piden consejos para determinadas situacio- 
Cuadro III

Protocolo de la entrevista EI.

Tarea introductoria: Clasificar

Finalidad

La finalidad de esta primera tarea es iniciar a los chicos en una situación de investigación y verificar modelos clasificando las fotos presentadas en grupos de la investigación.

\section{Instrucción}

a) Clasifica estas fotos en grupos: coloca juntas las que piensas que tienen algo en común y en otro grupo las que crees que son diferentes. Después intenta hacer algún otro grupo.

b) ¿Puedes explicar por qué tú agrupas los dibujos de esta forma?

c) ¿Puedes sugerir otras formas de agruparlos?

Tarea 1: Clasificar las fotos de acuerdo con situaciones donde se usan las matemáticas

Finalidad

Investigar las creencia acerca de qué son las matemáticas y buscar situaciones en las cuales la gente necesita usar matemáticas.

\section{Instrucción}

1.1. Clasifica las fotos de acuerdo con situaciones donde tú piensas que la gente necesita usar las matemáticas y con situaciones en las que tú piensas que no las necesitan.

1.2. ¿Por qué la gente necesita usar las matemáticas en este grupo de fotos?

1.3. ¿Por qué la gente no necesita usar las matemáticas en este grupo de fotos?

1.4. Ahora, para cada foto dime:

1.4.1. ¿Qué hace la gente?

1.4.2. ¿Qué lugar ocupa el trabajo para ellos?

1.4.3. ¿Qué tipo de matemáticas necesitan usar estas personas?

1.4.4. ¿Qué tipo de matemáticas son necesaria en esta situación?

1.4.5. ¿Dónde piensas que han aprendido ese tipo de matemáticas?

Tarea 2: La matemática en la experiencia escolar

\section{Finalidad}

Investigar las creencias de los chicos acerca de qué relaciones se establecen entre «fracaso» o «éxito» en la matemática escolar y el tipo de trabajo que la gente hace. Obtener datos del significado de las matemáticas en su experiencia escolar.

2.1. Sitúa a un lado las fotos en las que piensas que la gente ha tenido éxito en matemáticas y, al otro, aquéllas en las que te parezca que ha fracasado.

2.2. Da razones de la forma de agruparlas

2.3. ¿Quién piensas que era el mejor alumno de matemáticas en la escuela?

2.4. ¿Quién piensas que era el peor alumno de matemáticas en la escuela?

2.5. ¿Hay alguno de ellos que pienses que no ha ido nunca a la escuela?

Tarea 3: Importancia de la matemática y éxito y fracaso en matemáticas en la escuela

\section{Finalidad}

Investigar las creencias acerca de la importancia de la matemática y éxito o fracaso en matemáticas en la escuela. Obtener datos del significado de la matemática en su experiencia escolar.

\section{Situación}

Foto de clase con la profesora y los alumnos

3.1. Imagínate que es una clase de matemáticas ¿Piensas que es importante para ellos?

3.2. ¿Qué tipo de tema podría estar explicando la profesora? Explícame un poco qué es la matemática.

3.3. ¿Dónde piensas que pueden utilizar este tipo de conocimiento?

3.4. Lo que piensas que ellos aprenden, ¿tiene alguna importancia en el taller? ¿Por qué?

3.5. Si tú pudieras elegir, ¿qué te gustaría aprender en clase de matemáticas en la escuela?

3.6. Ahora pensemos en los alumnos. Algunos de ellos son muy buenos en matemáticas en la escuela. ¿Por qué?

3.7. Y algunos tienen dificultades ¿Por qué? ¿Dónde está el fallo?

3.8. ¿Qué piensas que se puede hacer para ayudar a un alumno que tiene dificultades?

3.9. ¿En tu opinión, ¿hay algo que el profesor puede hacer para ayudar a los alumnos con dificultades?

3.10. Ahora, cuéntame algo de ti. ¿Cómo te iba en la escuela? ¿Y con las matemáticas?

3.11. ¿Puedes recordar algún tema en el que te fuera bien en la escuela? ¿Y alguna ocasión en que tuvieras dificultades? ¿Qué clase de sentimientos asocias tú con cada una de estas situaciones? 
Tarea 4: Creencia de la importancia de la matemática en el taller. Conexiones entre taller y matemáticas.

\section{Situación}

Fotografía de gente trabajando en un taller de ebanistería

4.1. Pensemos en estos chicos. te para ellos?

4.2. Muchos de estos chicos han estado poco tiempo en la escuela: sin embargo, saben hacer cálculos muy bien. ¿Piensas que es posible? ¿Por qué?

4.3. ¿Piensas que lo que estos chicos aprenden en la calle y en el taller puede ayudarles en la escuela? ¿Cómo? ¿Los profesores de matemáticas aceptan esos conocimientos?

4.4. Piensa en estos chicos. ¿Qué aspiraciones y qué temores tienen paraencontrar trabajo? ¿Qué influye en esto? ¿Piensas que sería lo mismo si fuera otro trabajo?

4.5. Ahora cuéntame algo sobre ti. ¿Cómo te va en el taller? aprender en el taller?

4.6. De lo que te gusta hacer en el taller, ¿piensas que para ello necesitas aprender matemáticas? ¿Qué otro tipo de conocimientos (destrezas mentales)?

4.7. ¿Cuánto tiempo piensas que se necesita para ser un buen profesional?

4.8. ¿Puedes recordar algún proyecto en el que te fuera bien en el taller? ¿Y alguna ocasión en que tuvieras dificultades? ¿Qué clase de sentimientos asocias con cada una de estas situaciones? ¿Qué piensas que es importan¿Qué es lo que más te gusta
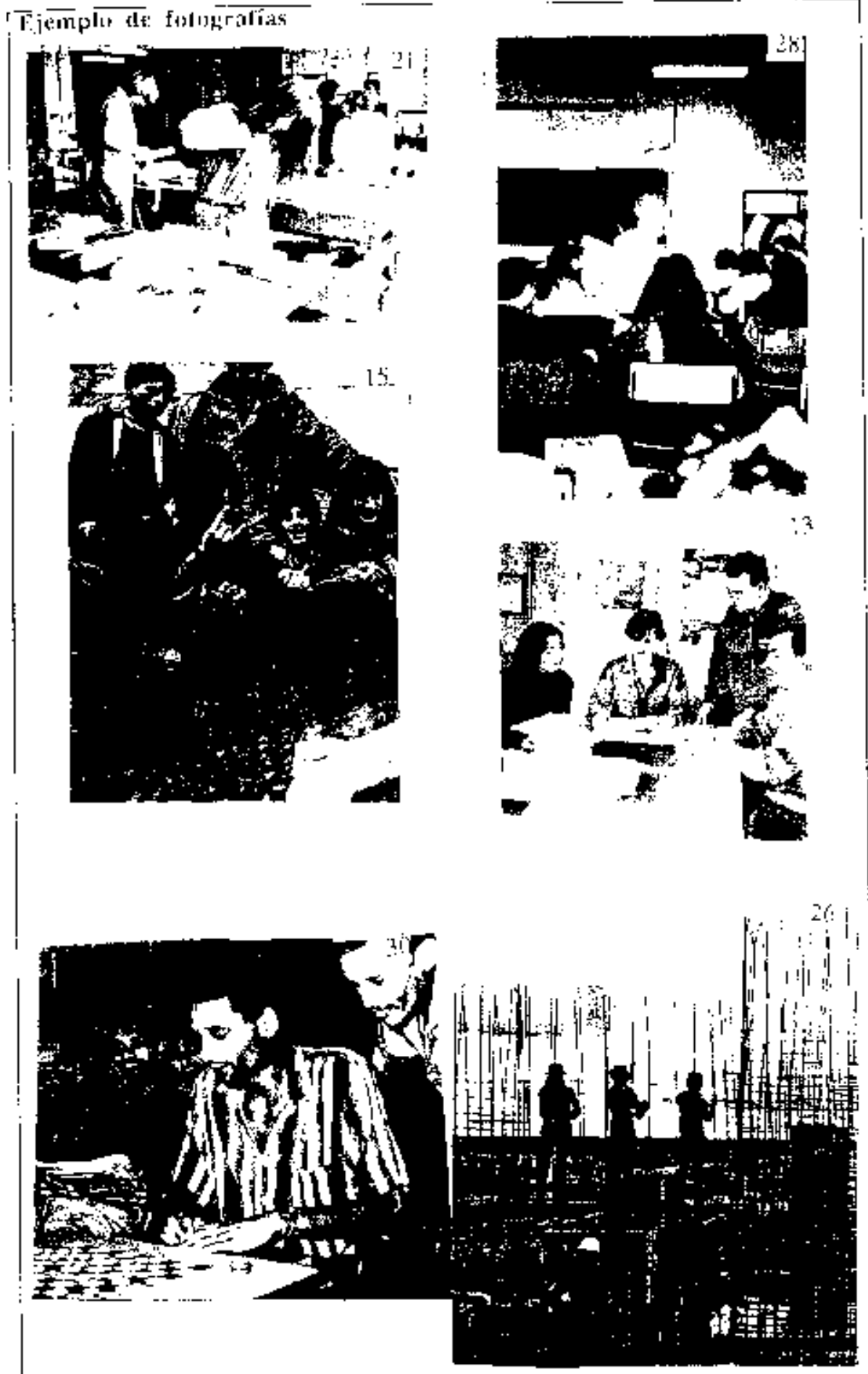
nes en las que participan jóvenes como ellos, etc. El entrevistado se proyecta en las elecciones que realiza. En definitiva, lo que se intenta es llegar a conocer, no únicamente cómo piensan estos jóvenes y de qué hechos viven, sino cómo proyectan creencias y reacciones emocionales sobre su mundo educativo y laboral.

Los objetivos al realizar está entrevista son:

A. Obtener datos y comprender.
1. La matemática en su experiencia escolar (emocióncreencia)

2. La matemática en su vida (hechos)

3. La matemática fuera del sistema escolar (creencia)

a) Taller y expectativa de trabajo

b) Taller y papel que juega la matemática

Cuadro IV

Mapa de humor de los problemas.

\section{EI tiempo}

\section{Menos fresco}

Predominarati los cielos despejados en in capital y su periferia, y los cielos nubosos en en la rierra ocoidental Ios vientos sopia rin flojos del Suroeste. ran flojos del Suroeste, pero moderados en la sierra. Habrá heladas de madrutgada, con formación de hiclo.en altitudes superiores a los 1.300 metros. Se formarán nieblas $y$ neblinats durante Ix mafiana. preferentemente en los vaites de ta sienra y cuencas del Jarama yded Manzanadels.

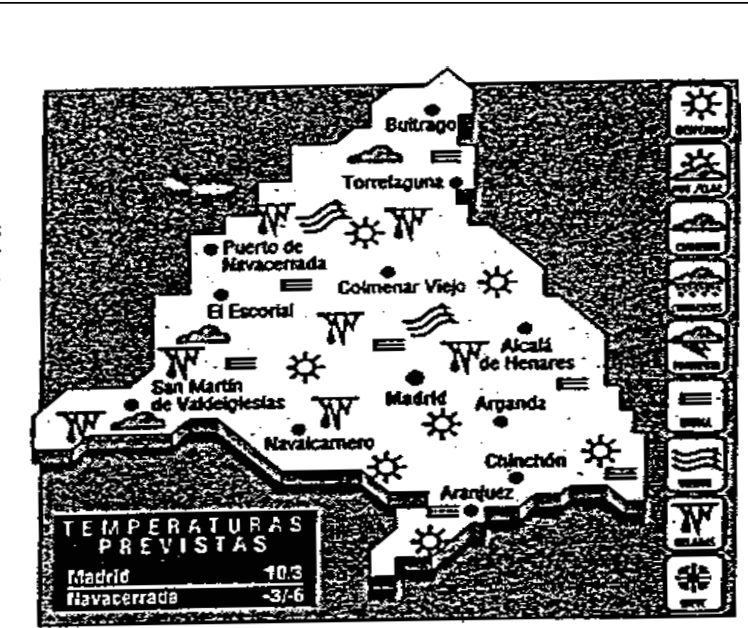

MAPA DE HUMOR DE LOS PROBLEMAS

¿Te has fijado alguna vez en los mapas del tiempo? Seguro que sí.

Aquí tienes un mapa de la provincia de Madrid en el que se pronostica el tiempo que hará a lo largo del día. Gracias a los símbolos que aparecen podemos saber que en Madrid-capital hace sol, que en el Escorial el cielo está cubierto, que en el Puerto de Navacerrada hay heladas, que en ningún sitio de la provincia de Madrid nevará, etc.

Pues algo parecido vamos hacer para cada una de las actividades que se te han propuesto: tienes que hacer TU MAPA DE HUMOR DE LOS PROBLEMAS. Observa que en la parte superior de la hoja de cada actividad encontrarás un cuadro con unos símbolos. Señala los que expresen cómo te has sentido tú al realizar el problema, indicando en qué momento del problema te encontrabas.

\begin{tabular}{|c|c|}
\hline Curiosidad $\approx$ & Desconcierto $\uparrow \rightarrow$ \\
\hline Animado dd & Come la cabeza 娄 \\
\hline Desesperación $\sigma$ & Gusto $\bullet$ \\
\hline Tranquilidad & Indiferencia $=$ \\
\hline Prisa $\lambda$ & Diversión $\}$ \\
\hline Aburrimiento 视 & Confianza \\
\hline De «abuty» $\mathrm{f}$ & Bloqueado \\
\hline
\end{tabular}


c) La matemática en un contexto de desventaja socioeconómica

B. Indagar si sus reacciones emocionales hacia la matemática van íntimamente ligadas o están fuertemente influenciadas por las convenciones culturales, por la representación social del conocimiento matemático y por la definición de su identidad social.

El material utilizado ha sido el siguiente: fotografías con personas en situaciones diferentes y que reflejan elementos de la cultura que antes habíamos observado (oposición entre lo formal y no formal, el grupo informal en el que están insertos, el mundo del trabajo, su significado, la división entre mental y manual).

Se seleccionaron 35 fotografías, que se agruparon del siguiente modo:

1. Grupo de trabajadores de tipo intelectual o de trabajo académico (corresponden a las fotos numeradas 2, 5, 11, $13,14,18,35)$

2. Grupo de trabajadores de tipo práctico o trabajo manual y juego (corresponden a los números $3,6,9,10$, $12,21,25,26,27,32)$

3. Grupos de estudiantes, bien en situación de estudio o de ámbito de diversión (números 4, 8, 15, 16, 17, 20, 23, $28,29,31,34)$

4. Grupos de personas que se encuentran en una situación de desventaja social y económica (en éstas incluía a gitanos y marroquíes) (números 1, 7, 15, 24, 30, 33)

\section{Tribus urbanas (número 22) (Cuadro III)}

Se realiza la entrevista a todos los alumnos de ebanistería que habían pasado por el centro durante los cursos 1994-95 y 1995-96 (un total de 23 personas), en la etapa inicial de entrada al centro, previa al momento inicial de la intervención. Se hacen entrevistas individuales, con una duración media de 45 minutos y máxima de una hora, siguiendo el protocolo.

Instrumentos de diagnóstico de las reacciones emocionales: el mapa de humor de los problemas

Como ya hemos indicado en este artículo, en el estudio de casos nos centramos, en primer lugar, en escenarios simples. Para determinar el origen de las reacciones emocionales del sujeto utilizamos la observación en clase y posterior confirmación en la entrevista y la propia autoevaluación del sujeto.

A continuación presentamos uno de los instrumentos de autoevaluación del sujeto, el mapa de humor de los problemas. Es un instrumento mediador válido para recoger información sobre las reacciones afectivas de los estudiantes y para favorecer el autoconocimiento de las reacciones emocionales de los alumnos y el control y regulación del aprendizaje.
El mapa de humor es un instrumento icónico que, a imitación de los mapas del tiempo, establece un código para expresar diferentes reacciones emocionales experimentadas por el estudiante en el transcurso de la actividad matemática. Viene siendo utilizado desde el curso 1994 en las clases de matemáticas de jóvenes que participan de una propuesta de formación profesional en la modalidad de talleres profesionales. Las emociones que aparecen registradas son consenso de las reacciones emocionales más relevantes de los estudiantes que se manifestaban en el aula, y tal como eran expresadas por este grupo de jóvenes (Cuadro IV). Fue elaborado por la investigadora-profesora después de examinar las reacciones emocionales y expresiones de los jóvenes de la investigación más usuales que verbalizaban las mismas y que habían sido recogidas durante un periodo largo de tiempo en las notas de campo, en las grabaciones de las sesiones, en los protocolos de los estudiantes y a través de otro instrumento utilizado para diagnosticar las reacciones emocionales (gráfica emocional), y en alguna sesión dedicada a trabajar de forma explícita la dimensión afectiva de que es «aprender a pensar positivamente».

Se aplica al grupo de jóvenes al terminar cada actividad matemática, quedando registrado en el protocolo de resolución mediante las anotaciones que realiza el individuo (Cuadro V).

En la investigación realizada se puso de manifiesto que este instrumento:

a) Favorece en el alumno el conocimiento propio de sus reacciones emocionales;

b) Favorece en el alumno el control y regulación del aprendizaje, pasando por el proceso metaafectivo de advertir, identificar, controlar y dar respuesta a la emoción;

c) Permite, al profesor, recoger información sobre las reacciones afectivas de los estudiantes (magnitud, dirección, consciencia y control de las emociones) y origen de las mismas (dinámica de interacción entre los factores afectivos y cognitivos);

d) Permite, al profesor, recoger información de las fases en las que se encuentran en la resolución de la tarea y los procesos cognitivos en que se trabajan.

\section{OTRAS FUENTES PARA LA RECOGIDA DE DATOS}

Hacemos notar que nuestro estudio tiene una intencionalidad didáctica con relación a la educación del afecto, a la alfabetización emocional. En el aula se estaba llevando a cabo un programa de actuación didáctica que tiene en cuenta la dimensión afectiva y de contexto de los sujetos (Gómez-Chacón, 1995a, 1995b, 1997a). Además de las entrevistas sobre situaciones y el mapa de 

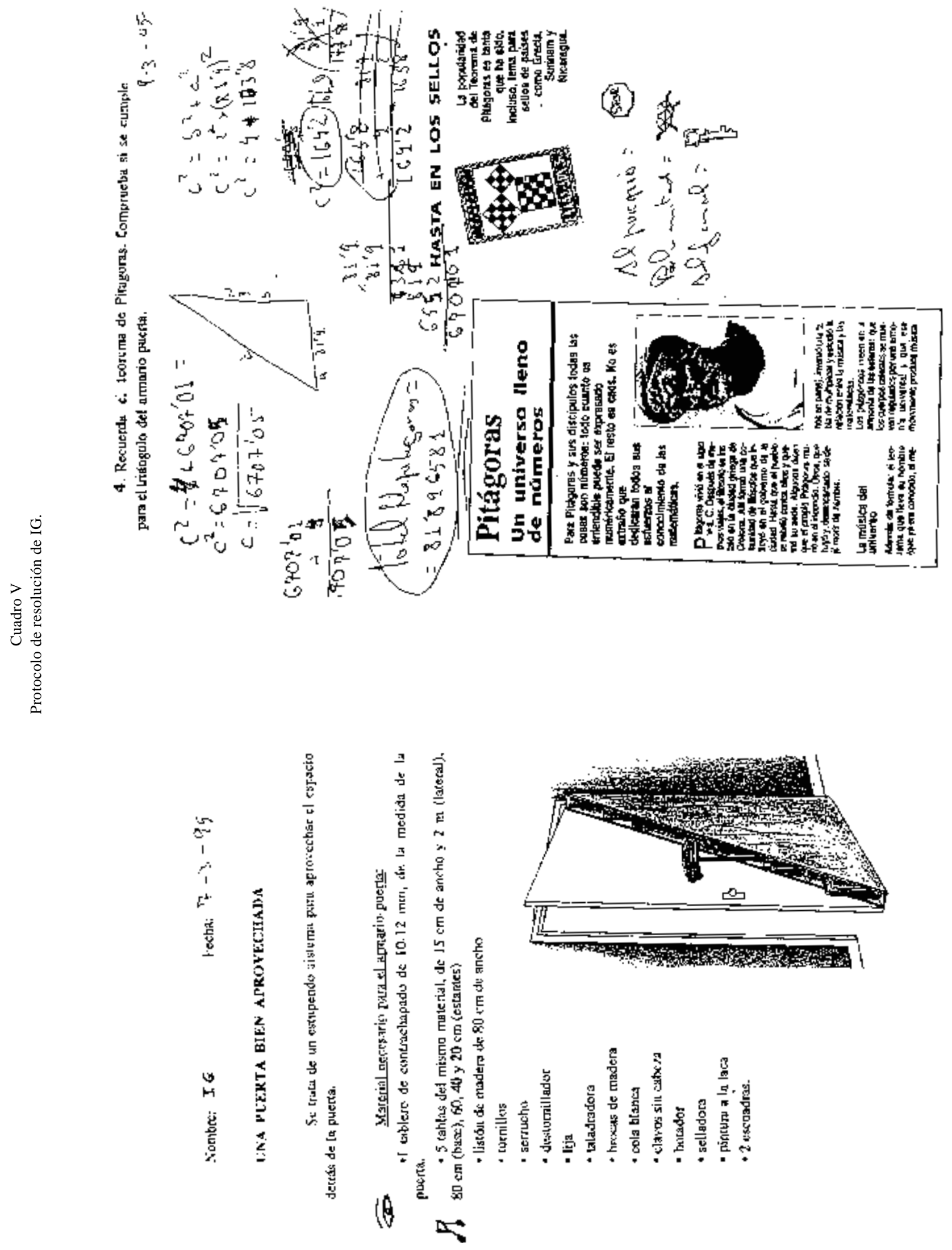
Figura 3

La figura muestra gráficamente la aplicación de los distintos procedimientos al grupo de jóvenes del estudio.

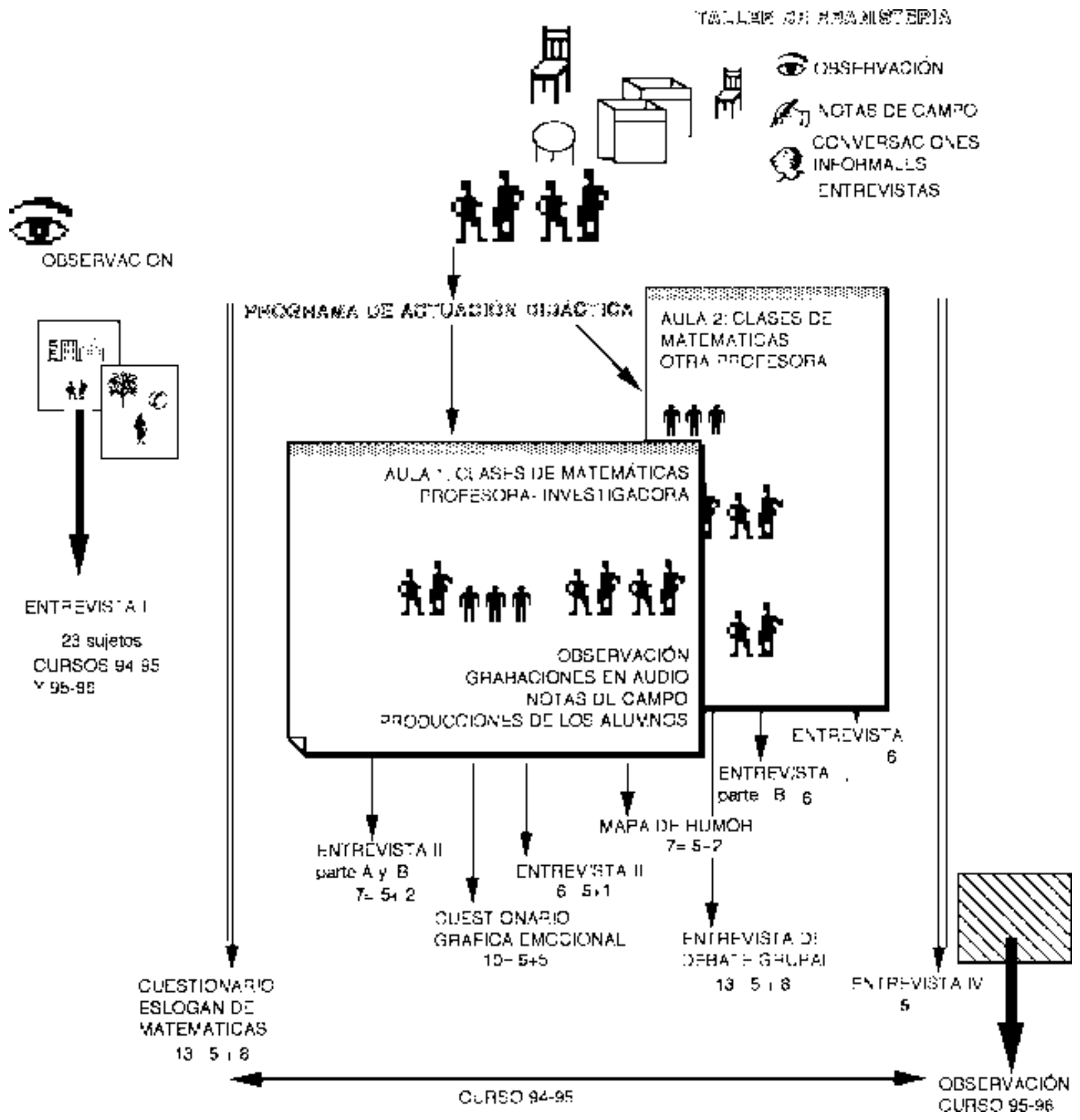

humor de los problemas descrito en el apartado anterior al grupo de estudio, se realizaron distintas entrevistas de valoración, regulación y utilización de la emoción y observación participante de las sesiones de aula recogi- das en grabaciones o notas de campo y las producciones de los alumnos. El mejor modo de visualizar cómo fueron aplicadas las pruebas al grupo de estudio es la figura siguiente (Fig. 3). 


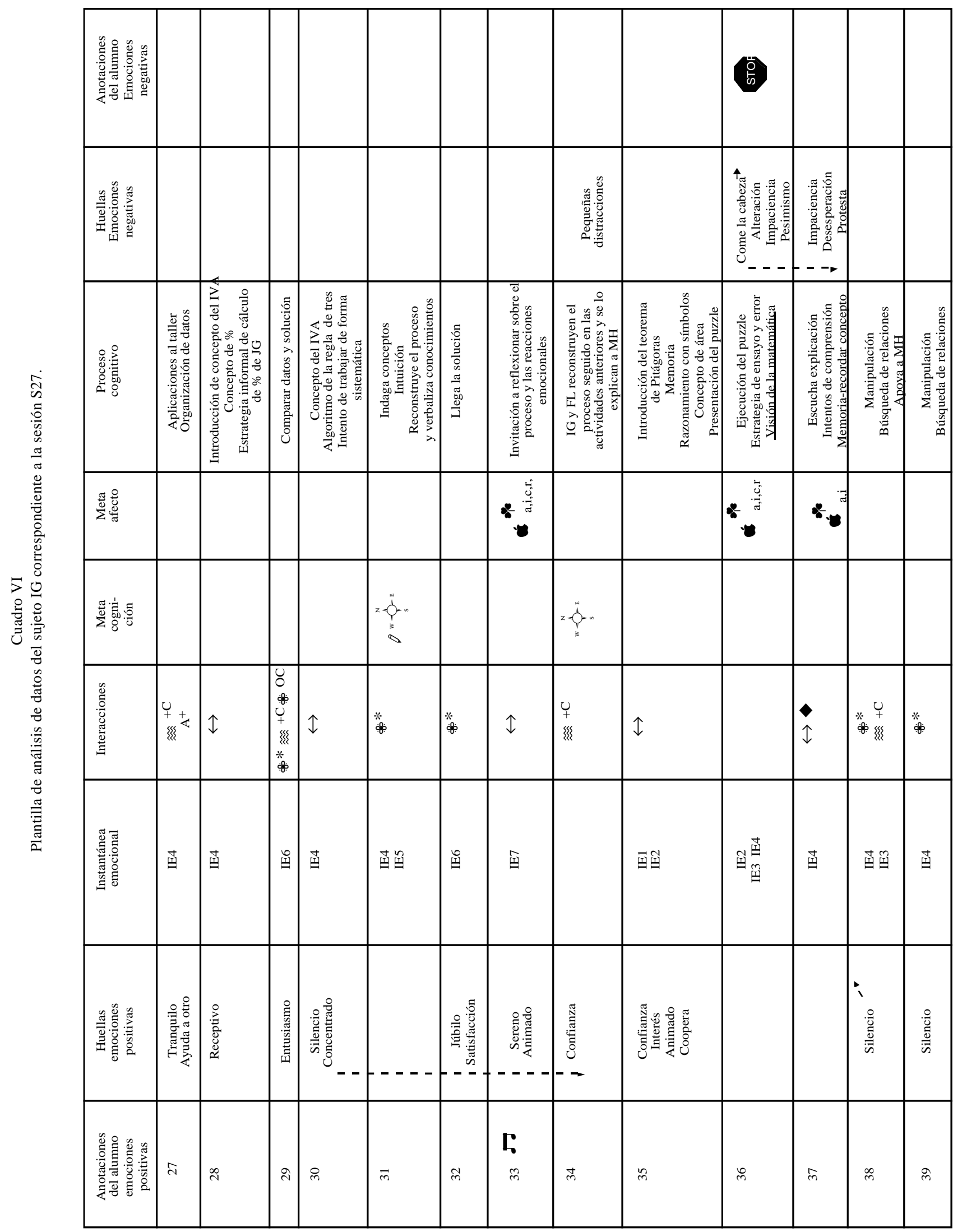


Podemos observar los dos ámbitos en los que se ha recabado la información, los procedimientos y número de sujetos. La variación del número ha estado determinada por la estructura de la investigación y condicionada a la movilidad de los sujetos.

\section{MÉTODO DE ANÁLISIS}

Nuestro enfoque en relación con los datos se ha orientado hacia el desarrollo de una comprensión en profundi- dad de los escenarios y los actores que participan en el estudio.

En la investigación cualitativa, el análisis de los datos se realiza durante todo el proceso. Los momentos de recogida y análisis de datos suelen alternarse y aparecen interconectados (Taylor y Bodgan, 1984; Huberman y Miles, 1994); es un proceso dinámico y creativo. En nuestro caso, el análisis de las observaciones del taller y de la entrevista primera proporcionó criterios para la elaboración del programa de actuación didáctica. El

Extracto de la sesión de aula y elementos recogidos por la investigadora para el análisis.

\section{Extracto de la grabación de una sesión de aula}

\section{URE 36-IG}

JG:- ¿Qué es eso?

IG:- Una cosa que te come la cabeza. (Está tratando de hacerse con el problema.)

FL:- Son piezas de un puzzle.

PR:- MH, ¿has visto como va?

MH:- No, no sé yo... esto como va, esto es más raro...

(No abandonan, protestan un poco.)

PR:- A ver, $\mathrm{MH}$, ¿cuántas piezas necesitas tú para hacer este armario? ¿Cuántas piezas grandes?

MH:- Dos.

PR:- ¿Cuántas baldas tienes?

MH:- Cinco.

PR:- Pues, mira, a ver qué medidas tienen...

IG:- Esto no sale, me estoy comiendo la cabeza, ;esto es un «comecabezas»! (Su tono de voz se va alterando, comienza a impacientarse, a verlo con pesimismo. Intenta con nuevas ideas, trata de encontrar otras formas; no abandona.)

FL:- A mí no me has dado esa hoja. (Se refiere a la del mapa de humor. La pide con insistencia.) ¿El triangulo no se puede poner?

PR:- A ver, ¿cómo que el triángulo no se puede poner? ¿Qué te dice el teorema?

FL:- Que $a^{2}+b^{2}=c^{2}$

MH:- (Interrumpe.) Esto, ¿qué es? ¿Cola?

FL:- Es cola o barniz, tú tienes que poner el proceso.

\section{URE 37-IG}

IG:- (Intenta pero no le sale.) ¡Me «caguen» la puta! (Comienza a desesperarse, a impacientarse cada vez más, pero continúa intentándolo.)

PR:- FL, Pitágoras, ¿qué nos decía: Si yo tengo un triángulo rectángulo... $a^{2}+b^{2}=c^{2}$. ¿Qué significaba esto? (Lo explico de nuevo en la pizarra para todos, me sorprende pero están de lo más atentos, todos en silencio). Comprueba.

JG:- A ver, señorita, explícamelo a mí. (Quiere que se lo repita a él en su papel, disposición receptiva, intentos de comprender.)

IG:- Vaya comedura de cabeza. (Continúa protestando; está inquieto, con un poco de desánimo, pues no le sale, pero parece estar atento a la explicación de la pizarra.)
Elementos recogidos por la investigadora para el análisis

\section{URE 36}

Este episodio emocional es señalado por el sujeto en el código del mapa de humor mediante Bloqueado, las huellas de emoción recogidas por la investigadora son de alteración, impaciencia, pesimismo, se come la cabeza (expresión del sujeto).

Con relación al metaafecto (en lo referente a la gestión de la actividad emocional del sujeto) es consciente, regula la emoción, advierte, identifica, controla a través de la expresión mediante el mapa de humor, dibujándolo y expresándolo a la profesora. Da respuesta mediante la reflexión sobre su reacción emocional para utilizarla en un futuro. No abandona protesta.

Durante este episodio emocional, los procesos cognitivos, es decir, las exigencias cognitivas son las requeridas para la tarea de realización de un puzzle, para trabajar el teorema de Pitágoras, trabajando sobre la estrategia de ensayo y error.

El sujeto expresa su creencia de que la matemática es algo que le come la cabeza.

El sujeto trata de hacerse con el problema, su atención está concentrada en el problema (instantánea IE2, entrando en materia), y, aunque le cuesta seguir avanzando, le entran ganas de aparcar el problema (IE4, continuar avanzando). Intenta una búsqueda de nuevas ideas, de encontrar otras formas de colocar las piezas, de un planteamiento alternativo (IE3, fermentando).

URE 37

Sigue avanzando (IE4), permanece constante, pero parece tener la sensación de una complicación injustificada, se impacienta, protesta, desespera. (Son huellas tomadas por la investigadora.)

Recibe el soporte cognitivo de la profesora sobre el teorema de Pitágoras, que la profesora explica en la pizarra. Avanza con el apoyo de la profesora. (Esto se refleja en las interacciones.) En relación con la gestión de la actividad emocional, es consciente, advierte lo que le pasa, identifica, pero no controla. 
análisis de las notas de campo, de las transcripciones de las sesiones de aula, del instrumento de la gráfica emocional sirvió para la elaboración de un nuevo instrumento para las reacciones emocionales, para tomar decisiones sobre el tipo de estudiantes en los que se centraría el estudio de casos, etc. A lo largo del análisis se ha tratado de obtener una comprensión más profunda de lo que se está estudiando y refinar de forma continuada nuestras interpretaciones.

El tratamiento de los datos se ha realizado con arreglo a los siguientes pasos:

1) Escucha y transcripción de las grabaciones de las entrevistas y de las sesiones de aula.

2) Lectura y relectura cuidadosa del material dejándonos impregnar por la información e identificando tendencias y patrones relevantes. Determinar una estructura temática y arquitectónica del análisis que nos permita armonizar los distintos niveles de la estructura de la investigación.

3) Delimitación de temas. Los temas que vertebraron el análisis, enunciados inductivamente a partir de las tendencias y patrones, fueron:

- El mundo y la identidad de los jóvenes de la investigación.

- Qué es aprender y saber matemáticas.

- Diagnóstico de la interrelación entre cognición y afecto en matemáticas.

- Rutas significativas que parece seguir la interacción entre cognición y afecto.

4) Análisis. Se selecciona la información necesaria para cada tema

Esto conlleva:

a) delimitación progresiva del foco de estudio;

b) agrupar datos cuantitativos, porcentajes, frecuencias;

c) realización de esquemas y diagramas como modo de sintetizar la información;

d) elaboración de volcados en algunos casos para el análisis de los datos:

- definición de unidades de análisis significativas;

- categorías;

- por último, comprender los datos en el contexto en que fueron recogidos.

e) selección de extractos de los protocolos para dar ejemplos y para el estudio de casos usados a través del análisis.
Pasamos a continuación a explicitar las categorías utilizadas y comentamos un ejemplo de los volcados (Cuadro VI) y de estas categorías en el análisis. Tomaremos el correspondiente a uno de los episodios emocionales de los sujetos del estudio de casos IG, y señalaremos la explicación a distintos episodios emocionales (unidades de reacción emocional URE, cuadro VII).

Describimos las categorías siguientes:

- Anotaciones del alumno de emociones positivas y negativas. Son las marcas que deja el alumno registradas sobre sus reacciones emocionales positivas o negativas en el proceso de resolución de la actividad matemática. Están extraídas, en este caso que presentamos, del mapa de humor.

- Huellas de emociones positivas y negativas. Son las señales que ha recogido la profesora durante la sesión de aula o las que en análisis de la sesión mediante la grabación y las notas de campo se evidencian y permiten describir, corroborar la emoción del estudiante.

- Instantáneas emocionales. Son «fotografías» de los estados característicos, elementos relevantes que tienen lugar mientras discurre el razonamiento del estudiante, momentos claves en los que se articula afecto y cognición. Están establecidas desde la concepción de las fases y proceso de resolución de problemas, pretenden describir «estados psicológicos» del estudiante (Mason, Burton y Stacey, 1982). En esta categoría, la investigadora recoge lo que se percibe, identifica y expresa en el documento de la sesión de aula -éste era resultado de la transcripción de la grabación de una sesión de aula completada con las notas de campo escritas por la investigadora bien en el transcurso de la sesión o después de la misma-). Distinguimos las siguientes instantáneas emocionales: primeros contactos (IE1), entrando en materia (IE2), seguir avanzando (IE4), fermentando (IE3), mostrarse escéptico (IE5) y estado contemplativo (IE6).

- Proceso cognitivo. Exigencias cognitivas que son necesarias en el proceso de ejecución y aprendizaje de la actividad matemática en ese momento. Son explicitadas por la investigadora.

- Interacciones. En esta categoría se recogen las interrelaciones sociales (alumno-profesora, alumno-alumno, alumno-grupo, profesora-grupo) en las cuales se desarrolla el proceso de enseñanza y aprendizaje. Se recogen los beneficios y obstáculos tanto cognitivos como motivacionales (aportados por la profesora, los compañeros) que influyen en la construcción del conocimiento. En esta categoría la investigadora recoge lo que se percibe, identifica y expresa en el documento de la sesión de aula.

- Metacognición. Toma de conciencia de la actividad mental. En esta categoría vamos a considerar lo que en la literatura pertinente se viene considerando como gestión de la actividad mental -planificación, control y regulación-. En esta categoría, la investigadora recoge lo que se percibe, identifica y expresa en el documento de la sesión de aula y en las producciones de los sujetos. 
Cuadro VIII

Rutas significativas que parecen seguir la interacción del afecto y la cognición en el sujeto IG. Síntesis de las anotaciones del alumno y las observaciones de la investigación de la estructura afecto-cognición.

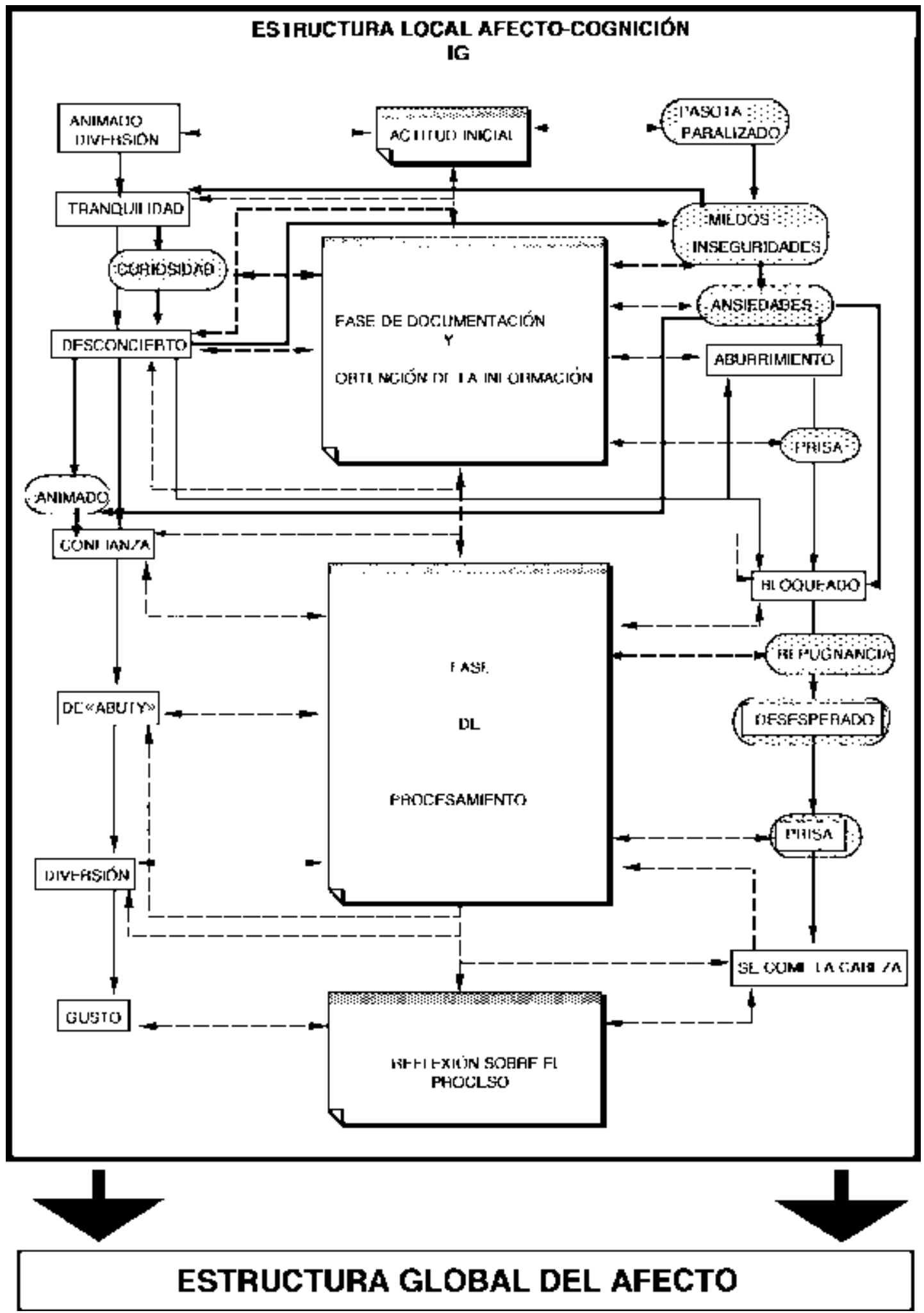


- Metaafecto. Toma de conciencia de la actividad emocional -valoración, expresión; regulación y utilización de la emoción-, dado que ha sido uno de los aspectos esenciales sobre los que se trataba de incidir en el programa de intervención. En esta categoría, la investigadora recoge lo que se percibe, identifica y expresa en el documento de la sesión de aula y en las producciones de los sujetos.

Para su representación en el análisis se han representado símbolos como:

$\leftrightarrow$ Intervención de la profesora, soporte afectivo.

惢 Interacción positiva hacia los otros, soporte cognitivo para los otros.

Regulación de la emoción, etc.

Paso a ejemplificar con la URE 36 y URE 37 cómo se realizó el análisis y cómo queda explicitado en el vaciado el estado emocional del sujeto IG en uno de los momentos correspondientes a la sesión de aula S27: «Una puerta bien aprovechada».

Los episodios de reacción emocional nos han permitido indagar algunas características o dimensiones del estado emocional de los sujetos: magnitud, dirección, nivel de de gestión de la actividad emocional (conciencia, control) e interacción con los procesos cognitivos.

Ayudándonos de estos análisis previos de las distintas sesiones de aula (48 sesiones como media por individuo) y teniendo como horizonte describir las rutas significativas que parecen seguir la interacción del afecto y la cognición en cada sujeto, para cada individuo del estudio de casos tratamos de dar respuesta a estas cuestiones:

- Ante la tarea, ¿cuál es la actitud afectiva inicial?

- ¿A qué se deben las interrupciones (los cortes o saltos) en la interacción afecto-cognición? ¿Cómo se articulan con el proceso de resolución de problemas?

- ¿Cuáles son las reacciones emocionales más frecuentes?

- ¿Cómo se pueden catalogar sus tendencias cognitivas $\mathrm{y}$ afectivas?

Toda esta información, síntesis de las anotaciones del alumno y de las observaciones de la investigadora, nos permitió llegar a elaborar el perfil de cada sujeto, el mapa afecto-cognición. En este mapa queda reflejada la estructura local y global del afecto expresada a través de las emociones consensuadas en el mapa de humor, y las rutas de interacción con los procesos cognitivos (exigencias cognitivas). El cuadro VIII muestra el mapa afectocognición del sujeto IG.

Por último, hacemos notar que, con respecto a los elementos de rigor exigibles en el enfoque cualitativo de la investigación, a lo largo de todo el proceso hemos ido reflexionando sobre nuestra acción y contrastándolo con distintos jueces llegando a consenso y en diferentes momentos. Destacamos los siguientes momentos: la validación del programa de actuación didáctica; el diseño de los instrumentos de recogida de datos; y la secuencia de análisis de los datos, tanto en el proceso de definición de unidades de análisis, temas y categorización como una vez realizado el análisis.

\section{RESUMEN}

En relación con los aspectos metodológicos de la investigación que presentamos en este artículo, las conclusiones pueden resumirse como sigue:

- El diseño metodológico ha resultado adecuado para cubrir el objetivo de nuestra investigación.

- Los instrumentos de recogida de datos diseñados expresamente para este trabajo han resultado ser una aportación determinante para el mismo, dada la escasez y falta de adecuación de instrumentos para poblaciones semejantes a la nuestra.

De éstos destacamos:

- El mapa de humor, que ha constituido un instrumento mediador válido para recoger información sobre las reacciones afectivas de los estudiantes. Ha favorecido el autoconocimiento de las reacciones emocionales de los alumnos y el control y regulación del aprendizaje.

- Las entrevistas diseñadas para poner de manifiesto creencias, emociones y la identidad de los jóvenes.

- La propuesta de inclusión de aspectos del mundo y la identidad de los jóvenes (escenarios complejos) en la metodología de la investigación ha sido un acierto de cara a la comprensión del afecto global del sujeto.

- Las categorías de análisis elaboradas han resultado eficaces para que los datos adquirieran sentido.

- El sistema de notación adoptado para representar las distintas categorías de análisis ha facilitado, en gran manera, el reconocimiento de patrones y la comparación de los datos que los sujetos apuntaban.

La aproximación mediante escenarios simples nos ha llevado a una serie de conclusiones, de las que destacamos las siguientes:

- En el estudio de casos se pudo constatar que existen tendencias y perfiles que permiten delimitar las características del afecto global del sujeto. Los datos del estudio de casos apuntan dos rutas que implican al afecto y a la cognición; una que podríamos llamar positiva y otra, negativa. A través de ellas se ha podido delimitar un «modelo» para el sujeto. Se ha podido constatar que, entre las características que comparten la dimensión local del afecto de cada individuo, aparecían reiteradas 
formas de reaccionar ante la matemática y su aprendizaje, coincidencias en su visión de la matemática, miedos inseguridades, evocaciones de la experiencia escolar, etc.

- Sin embargo, se observa que las evoluciones de los sujetos eran diferentes. Tras aparentes coincidencias en la interrelación entre cognición y afecto se daba una fuerte diferencia y ésta estaba estrechamente ligada a sus posicionamientos como miembros de un grupo social, que configura su afecto global y que está incidiendo en el afecto local. Por tanto, para comprender la dimensión afectiva del estudiante en relación con la matemática es necesario tener en cuenta la dimensión afectiva del individuo en escenarios más complejos que permiten contextualizar las reacciones emocionales en la realidad social que las produce.

- La identidad social de los jóvenes contribuye a configurar, por su carácter relacional y dinámico, la estructura local y global del afecto; es decir, incide en las rutas del afecto local que se establecen con el sistema cognitivo y contribuyen a la construcción de estructuras generales del concepto de uno mismo, y a la formación de creencias acerca de la matemática y su aprendizaje.

- Los datos ponen de manifiesto que los estudiantes reciben continuos mensajes del entorno sobre qué significa conocer matemáticas y cuál es el significado social de su aprendizaje. La estructura de autoconcepto como aprendiz de matemáticas está relacionada con sus actitudes, con la perspectiva en que sitúa el mundo matemático y con su identidad social.

\section{REFERENCIAS BIBLIOGRÁFICAS}

ABREU, G. (1993). The relationship between home and school mathematics in a farming community in rural Brazil. Tesis doctoral. Universidad de Cambridge.

BISHOP, A. J. (1988). Mathematical Enculturation: A Cultural Perspective on Mathematics Education. Dordrecht: Kluwer.

BISHOP, A. J. y ABREU, G. (1991). Children's use of outsideschool knowledge to solve mathematics problems in school, en Furinghetti, F. (ed.), Proceedings of the Fifteenth International Conference for the Psychology of Mathematics Educaction. Vol. I, pp. 128-135. Asís: Italia.

CAMILLERI, C., KASTERSZTEIN, J., LIPIANSKY, E.M., MALESWSKA-PEYRE, H., TABOADA-LEONETTI, I., VASQUEZ, A. (1990). Stratégies identitaires. París: Presses Universitaires de France.
- La inclusión de la perspectiva de la identidad social acentúa la necesidad de considerar la influencia de las relaciones simbólicas sociales. Es en este nivel donde hemos tratado de comprender cómo las valoraciones, a las cuales los grupos sociales ligan las diferentes formas de conocimiento, son mediadoras en la cognición matemática. La atención a este aspecto nos ha proporcionado datos para construir una interpretación global del afecto en cada sujeto.

\section{AGRADECIMIENTOS}

Mi agradecimiento a Miguel de Guzmán, Rosa Aparicio, R. Gutiérrez, M.L. Mata, por el interesante intercambio de ideas recibido en el transcurso de esta investigación.

\section{NOTAS}

1 No pretendemos dar una panorámica exhaustiva de estudios sobre afectividad en educación matemática. En el presente artículo nos limitaremos a la opción elegida en la investigación.

${ }^{2}$ Hablar sobre un escenario es hablar sobre todo aquello que organiza una escena, de lo que se está poniendo en juego en un ámbito y en un tiempo concreto, con unos recursos determinados.

${ }^{3}$ Entendidos como las exigencias cognitivas que son necesarias en el proceso de ejecución y la actividad matemática en ese momento.

${ }^{4}$ La creencia en uno mismo como buen (o mal) resolutor de problemas; la expectativa de éxito o fracaso ante un problema matemático, y la anticipación de sentimientos, emociones al comenzar o en el transcurso al final de la actividad matemática, la identidad social, etc.
CARRAHER, T. N., CARAHER, D.W.y SCHLIEMANN, A. D. (1988). Na Vida, Dez; Na Escola Zero. Os Contextos Culturais da Aprendizagem da Matemática. São Paulo: Cortez.

CIVIL, M. (1994). Connecting the home and the school: funds of knowledge for mathematics teaching and learning. AERA Annual Meeting: Everyday mathematics: What is it and What can schools do about it? New Orleans.

COBB, P., YACKEL, E. y WOOD, T. (1989). Young childrens's emotional acts while engaged in mathematical problem solving, en McLeod, D.B. y Adams, V.M. (eds.), Affect and mathematical problem solving: A new perspective, pp. 117148. Nueva York: Springer Verlag. 
COBB, P., YACKEL, E. y WOOD, T. (1992). A constructivist alternative to the representational view of mind in mathematics education. Journal for Research in Mathematics Education, 23, pp. 2-23.

COBB, P., WOOD, T. y YACKEL, E. (1991). A constructivist approach to second grade mathematics, en Glasersfeld, E. von (ed.), Radical constructivism in mathematics education, pp. 157-176. Dordrecht, the Netherlands: Kluwer Academic Publishers.

CONFREY, J. (1991). What constructivism implies for teaching, en Davis, R.B., Maher, C. A. y Noddings, N. (eds.), Constructivist views on teaching and learning mathematics, p. 107-122. Reston, VA: National Council of Teachers of Mathematics.

D'AMBROSIO, U. (1985). Ethnomathematics and its place in the history and pedagogy of mathematics. For the Learning of Mathematics, 5 (1), pp. 44-48.

EISENHART, M. A. (1988). The ethnographic research tradition and mathematics education research. Journal for Research in Mathematics Education, 19(2), pp. 99-114.

GOLDIN, G. A. (1988). Affective representation and mathematical problem solving, en Behr, M.J. Lacampagne, C. B. y Wheler, M.M. (eds.), Proceedings of the Tenth Annual Meeting on the Psychology of Mathematics Education, North American Chapter of International Group, pp. 1-7. North Illinois University. DeKalb, IL.

GÓMEZ-CHACÓN, I. M. (1995a). Mathematics in the «Centrotaller»: Looking for the connections between the affective issues and the cultural influences in the mathematical learning. Documento presentado en 19thAnnual Meeting of International Group for the Psychology of Mathematics Education (PME) y publicado en Booklet Cultural aspests in the Learning of Mathematics, Some current developments, PME19, p. 33-46. Recife, julio de 1995.

GÓMEZ-CHACÓN, I. M. (1995b). Las matemáticas en los centros-taller: Cómo establecer un puente que una las matemáticas de dentro y fuera de la escuela. Herramientas. Revista de Formación para el Empleo, año VII, 6(42), pp. 32-38.

GÓMEZ-CHACÓN, I. M. (1997). Procesos de aprendizaje en matemáticas con poblaciones de fracaso escolar en contextos de exclusión social. Las influencias afectivas en el conocimiento de las matemáticas. Tesis doctoral. Universidad Complutense.

GÓMEZ-CHACÓN, I. M. (1997a). La alfabetización emocional en educación matemática: actitudes, emociones y creencias. Revista de Didáctica de las Matemáticas UNO, 13, pp. 7-22.

HUBERMAN, A. M. y MILES, M. (1994). Data management and analysis methods, en Denzin, N.K. y Lincoln, Y.S. (eds.), Handbook of qualitative research, pp. 428-444. Londres: Sage Publications.

LAFORTUNE, L. y ST-PIERRE, L. (1994). La pensée et les émotions en mathématiques. Métacognition et affectivité. Quebec: Les Editions Logiques.

LAVE, J. (1988) Cognition in practice. Cambridge: Cambridge University Press. Trad. de Botella, L., 1991. La cognición en la práctica. Cognición y desarrollo humano. Barcelona: Paidós.
LERMAN, S. (ed.) (1996). Socio-cultural approaches to mathematics teaching and learning, Educational Studies in Mathematics, 31(1-2). Monografía.

LLOYD, B. y DUVEEN, G. (1992). Gender identities and education: The impact of starting school. Londres: Harvester Wheatsheaf.

MANDLER, G. (1984). Mind and body: Psychology of emotion and stress. Nueva York: Norton.

MANDLER, G. (1989). Affect and learning: Causes and consequences of emotional interactions, en McLeody Adams, V.M. (eds.), Affect and mathematical problem solving: A new perspectitve, pp. 3-19. Nueva York: Springer-Verlag.

MASON, J., BURTON, L. y STACEY, K. (1982). Thinking mathematically. Londres: Addison-Wesley. Trad. de Martínez. M., 1988. Pensar matemáticamente. Barcelona: Labor.

McLEOD, D.B.(1988). Affective issues in mathematical problem solving: Some theoretical considerations. JournaI for Research in Mathematics Education, 19, pp. 134-141.

MCLEOD, D.B. (1990). Information-processing theories and mathematics learning: the role of affect. International Journal of Educational Research, 14, pp. 13-29.

MCLEOD, D.B. (1992). Research on affect in mathematics education: A reconceptualization, en Douglas Grows, A. (ed.), Handbook of Research on Mathematics Teaching and Learning, pp. 575-596. Nueva York: Macmillan, NCTM.

MCLEOD, D.B. (1994). Research on affect and mathematics learning in the JRME: 1970 to the present. Journal for Research in Mathematics Education, 25(6), pp. 637-647.

MELLIN-OLSEN, S. (1987). The politics of mathematics education. Boston: D. Reidel.

MOLL, L. (1992). Bilingual classroom studies and community analysis: some recent trends. Educational Researcher, 21(2), pp. 20-24.

NIMIER, J. (1993). Defence mechanisms against mathematics. For the Learning of mathematics, 13(1), pp. 30-34.

SCHOENFELD, A.H.(1991). On mathematics as sense-making: An informal attack on the unfortunate divorce of formal and informal mathematics, en Voss, J.F., Perkins, D.N. y Segal, J. (eds.), Informal reasoning and instruction, pp. 311-343. Hillsdale, NJ: Lawrence Erlbaum.

SCHOENFELD, A.H. (1994). A discourse on methods. Journal for Research in Mathematics Education, 25(6), pp. 697-710.

STEFFE, L.P. (1991). The constructivist teaching experiment: Illustrations and implications, en Glasersfeld, E. von (ed.). Radical constructivism in mathematics education, pp. 177194. Dordrecht, the Netherlands: Kluwer Academic Publishers.

STIGLER, L.W. y BARANES, R. (1988). Culture and mathematics learning, en Rothkopf, E.Z. (ed.), Review of Research in Education, Vol. 15, pp. 97-169.Washington, DC: American Educational Research Association.

TAYLOR, S. J. y BOGDAN, R. (1984). Introduction to qualitative research methods. The search for meanings. Nueva York: Jonh Wiley y Sons. Trad. cast. de Piatigorsky, J. Introducción a los métodos cualitativos de investigación. Barcelona: Paidós. Primera reimpresión, 1992. 